\title{
Density-functional Study of Small Molecules within the Krieger-Li-Iafrate Approximation
}

\author{
Yong-Hoon Kim, ${ }^{(a)}$ Martin Städele, ${ }^{(a, b)}$ and Richard M. Martin ${ }^{(a, c)}$ \\ Department of Physics, ${ }^{(a)}$ Beckman Institute for Advanced Science and Technology, ${ }^{(b)}$ and \\ Materials Research Laboratory, ${ }^{(c)}$ University of Illinois at Urbana-Champaign, Urbana, Illinois \\ 61801
}

(August 13, 2018)

\begin{abstract}
We report density-functional studies of several small molecules $\left(\mathrm{H}_{2}, \mathrm{~N}_{2}, \mathrm{CO}, \mathrm{H}_{2} \mathrm{O}\right.$, and $\left.\mathrm{CH}_{4}\right)$ within the Krieger-Li-Iafrate (KLI) approximation to the exact Kohn-Sham local exchange potential, using a threedimensional real-space finite-difference pseudopotential method. It is found that exchange-only KLI leads to markedly improved eigenvalue spectra compared to those obtained within the standard local-density approximation (LDA), the generalized gradient approximation (GGA), and the Hartree-Fock (HF) method. For structural properties, exchange-only KLI results are close to the corresponding HF values. We find that the addition of LDA or GGA correlation energy functionals to the KLI exact exchange energy functional does not lead to systematic improvements.
\end{abstract}

PACS numbers: 02.70Bf, 31.10.+z, 31.15.Ew, 31.15.Fx, 31.50.+w, 31.90+s 


\section{INTRODUCTION}

Density functional theory (DFT) within the Kohn-Sham (KS) single-particle formalism has now been established as a standard tool for the study of inhomogeneous quantum manyelectron systems. [1 3 3 The local density approximation (LDA) and generalized gradient approximation (GGA) are successful in describing the ground state properties of various systems. On the other hand, their eigenvalue spectra are known to be far from the reality, and it has been assumed that KS orbital eigenvalues are auxiliary quantities without any physical meaning except the highest occupied one. [3] However, it is clear that DFT is also a legitimate route to the study of excited state properties via perturbation theory [4] or time-dependent DFT. [5:6] Especially, Görling has recently formulated a KS formalism for excited states based on DFT perturbation theory and demonstrated that the KS eigenvalue difference is a well-defined zeroth order approximation to excitation energies. [7] This has been also explicitly confirmed for several atoms by accurate quantum Monte-Carlo (QMC) calculations. 8,9]

It appears that - at least for localized systems - the unrealistic description of eigenvalue spectra is mainly due to the deficiencies of the conventional approximations for the exchangecorrelation energy functional such as the LDA or GGA rather than the KS scheme itself. The origin of these deficiencies is now more or less understood from the investigations of the exact properties of the KS exchange-correlation potential $V_{x c}^{K S}$ which the LDA or GGA does not satisfy: (i) $V_{x c}^{K S}(\mathbf{r}) \rightarrow-1 / r$ as $r \rightarrow \infty$ for finite systems, [10,11] (ii) the eigenvalue of the highest occupied single particle states is the negative of the ionization energy, [10,12 (iii) $V_{x c}^{K S}(\mathbf{r})$ is self-interaction free, and (iv) $V_{x c}^{K S}(\mathbf{r})$ exhibits an integer discontinuity upon the addition of an electron. 13,14] However, it has been recognized that approximations to the local KS potential obtained from the orbital dependent exact exchange energy functional through the so-called optimized effective potential (OEP) method [15, 16] closely observe these conditions. [17, 18]

In spite of these good features, the implementation of the OEP method is very complicated and its computational cost is excessively high. Only spherical atoms, 16, 17, 19 jellium surfaces, [20] jellium spheres, [21] and periodic solids with a small unit cell [22] have been extensively studied, and there exists no investigation of molecular systems with the OEP method. However, about a decade ago, Krieger, Li, and Iafrate (KLI) proposed an approximation scheme to the OEP, and have shown that the KLI potential with the exact exchange-only energy functional (KLI-x) satisfies the above mentioned properties (i)-(iv)

of $V_{x c}^{K S}$, and accurately reproduces the OEP-x results for spherical atoms. [18] Within the KLI-x approximation, the workload in constructing the potential is significantly reduced, but this method is still relatively complicated compared with the LDA or GGA and studies are therefore limited. Atomic systems have been originally investigated by KLI, [18] and more recently by Grabo et al. 23] Bylander and Kleinman studied semiconductors with the KLI potential. [24] Although molecules are a very good testing ground of any functional approximation, only some diatomic molecules have been investigated by Grabo et al. [23]

In this article, we report a study of several closed shell diatomic and polyatomic molecules within the KLI scheme. The questions we address are the performance of the KLI approximation to the OEP with the exact exchange-only functional (KLI-x) and supplemented by LDA and Perdew-Burke-Ernzerhof GGA 25] correlation energy functionals (KLI-x+LDA-c 
and KLI-x+GGA-c) for both structural properties and eigenvalue spectra. The comparison with and qualitative differences from the LDA/GGA and the Hartree-Fock (HF) method will be emphasized. In addition, since we incorporate the exact exchange, we can examine the accuracy of the LDA and GGA correlation energy functionals. The underlying computational algorithm is the three-dimensional (3D) finite-difference pseudopotential method [26,27] and, to our knowledge, this is the first 3D KLI investigation of molecular systems.

The organization of the paper is as follows. In Sec. II, the formulation of KLI is reviewed and the differences of the KLI-x from the HF method will be emphasized. The implementation of KLI-x into our molecular code and its test results are described. In Sec. III, results and discussions for several molecules will be presented for both structural properties and eigenvalue spectra. Especially, comparisons between the KLI-x and the LDA/GGA or HF method are made, and physical origins of these differences will be discussed. In Sec. IV, we conclude the paper by summarizing our work and future prospects.

\section{FORMALISM}

\section{A. Approximation of OEP by Krieger, Li, and Iafrate}

We start from the spin-dependent KS equations for KS orbitals $\psi_{\sigma i}(\mathbf{r})$ for spin channel $\sigma=\uparrow, \downarrow$ and KS eigenvalues $\epsilon_{\sigma i}$, with the possibility of fractional occupation numbers $f_{\sigma i}$ (Hartree atomic units are used throughout the paper)

$$
\left[-\frac{1}{2} \nabla^{2}+V_{\sigma}^{K S}(\mathbf{r})\right] \psi_{\sigma i}(\mathbf{r})=\epsilon_{\sigma i} \psi_{\sigma i}(\mathbf{r})
$$

with the spin density

$$
n_{\sigma}(\mathbf{r})=\sum_{\mathrm{l}=1}^{N_{\sigma}} f_{\sigma i}\left|\psi_{\sigma i}(\mathbf{r})\right|^{2},
$$

where $N_{\sigma}$ is the number of occupied $\sigma$ spin orbitals. The effective KS potential $V_{\sigma}^{K S}(\mathbf{r})$ is composed of external, Hartree, and exchange-correlation contributions,

$$
V_{\sigma}^{K S}(\mathbf{r})=V_{e x t}(\mathbf{r})+V_{H}(\mathbf{r})+V_{x c, \sigma}^{K S}(\mathbf{r})
$$

where the exchange-correlation potential $V_{x c, \sigma}^{K S}(\mathbf{r})$ is given by

$$
V_{x c, \sigma}^{K S}(\mathbf{r})=\frac{\delta E_{x c}\left[\left\{n_{\uparrow}, n_{\downarrow}\right\}\right]}{\delta n_{\sigma}(\mathbf{r})} .
$$

If the exchange-correlation functional is explicitly orbital-dependent $E_{x c}\left[\left\{n_{\uparrow}, n_{\downarrow}\right\}\right] \equiv$ $E_{x c}\left[\left\{\psi_{\sigma i}\right\}\right]$, the corresponding $V_{x c, \sigma}^{K S}(\mathbf{r})=V_{x c, \sigma}^{O E P}(\mathbf{r})$ can be obtained by the OEP integral equation [15,16]

$$
\sum_{\sigma i} f_{\sigma i} \int d \mathbf{r}^{\prime}\left[V_{x c, \sigma}^{O E P}\left(\mathbf{r}^{\prime}\right)-u_{x c, \sigma i}\left(\mathbf{r}^{\prime}\right)\right] \psi_{\sigma i}^{*}\left(\mathbf{r}^{\prime}\right) G_{\sigma i}\left(\mathbf{r}^{\prime}, \mathbf{r}\right) \psi_{\sigma i}(\mathbf{r})+\text { c.c. }=0,
$$

where the quantity $u_{x c, \sigma i}(\mathbf{r})$ is given by 


$$
u_{x c, \sigma i}(\mathbf{r}) \equiv \frac{1}{f_{\sigma i} \psi_{\sigma i}(\mathbf{r})} \frac{\delta E_{x c}\left[\left\{\psi_{\sigma i}\right\}\right]}{\delta \psi_{\sigma i}^{*}(\mathbf{r})} .
$$

The KLI approximation to the OEP is obtained by ignoring certain terms whose average over the ground-state spin density vanishes. [18] It can be also heuristically derived by approximating $\left(\epsilon_{\sigma j}-\epsilon_{\sigma i}\right)^{-1}$, appearing in the Green's function in Eq. (5) by a constant $\Delta \epsilon_{\sigma}: 18$

$$
G_{\sigma i}\left(\mathbf{r}^{\prime}, \mathbf{r}\right) \equiv \sum_{j \neq i} \frac{\psi_{\sigma j}\left(\mathbf{r}^{\prime}\right) \psi_{\sigma j}^{*}(\mathbf{r})}{\epsilon_{\sigma j}-\epsilon_{\sigma i}} \approx \frac{\sum_{j \neq i} \psi_{\sigma j}\left(\mathbf{r}^{\prime}\right) \psi_{\sigma j}^{*}(\mathbf{r})}{\Delta \epsilon_{\sigma}}
$$

in which case Eq. (17) reduces to integrals over the wave functions independent of the spectrum. Slater's approximation [28] can be obtained by simplifying the numerator of Eq. (17) further to $\sum_{j \neq i} \psi_{\sigma j}\left(\mathbf{r}^{\prime}\right) \psi_{\sigma j}^{*}(\mathbf{r}) \approx \delta\left(\mathbf{r}-\mathbf{r}^{\prime}\right)$, thus ignoring $-\psi_{\sigma i}\left(\mathbf{r}^{\prime}\right) \psi_{\sigma i}^{*}(\mathbf{r})$ term. 15.18. Although the KLI approximation is generally applicable to any orbital dependent exchangecorrelation energy functional, we will concentrate on the case of the exact exchange energy functional defined as

$$
E_{x}\left[\left\{\psi_{\sigma i}\right\}\right]=-\frac{1}{2} \sum_{\sigma=\uparrow, \downarrow} \sum_{i, j}^{N_{\sigma}} f_{\sigma i} f_{\sigma j} \int d \mathbf{r} \int d \mathbf{r}^{\prime} \frac{\psi_{\sigma i}^{*}(\mathbf{r}) \psi_{\sigma j}^{*}\left(\mathbf{r}^{\prime}\right) \psi_{\sigma i}\left(\mathbf{r}^{\prime}\right) \psi_{\sigma j}(\mathbf{r})}{\left|\mathbf{r}-\mathbf{r}^{\prime}\right|},
$$

which has been actually used in this work. Here note that $\left\{\psi_{\sigma i}\right\}$ are KS orbitals rather than HF orbitals. In this case, $u_{x, \sigma i}(\mathbf{r})$ is just the HF local exchange potential expression evaluated with KS (instead of HF) orbitals $\left\{\psi_{\sigma i}\right\}$

$$
u_{x, \sigma i}(\mathbf{r})=-\frac{1}{\psi_{\sigma i}(\mathbf{r})} \sum_{j=1}^{N_{\sigma}} f_{\sigma j} \psi_{\sigma j}(\mathbf{r}) K_{\sigma j i}(\mathbf{r})
$$

where

$$
K_{\sigma j i}(\mathbf{r})=\int d \mathbf{r}^{\prime} \frac{\psi_{\sigma j}^{*}\left(\mathbf{r}^{\prime}\right) \psi_{\sigma i}\left(\mathbf{r}^{\prime}\right)}{\left|\mathbf{r}-\mathbf{r}^{\prime}\right|}
$$

The approximation of Eq. (17) leads to the KLI-x potential

$$
V_{x, \sigma}^{K L I-x}(\mathbf{r})=V_{x, \sigma}^{S}(\mathbf{r})+\sum_{i=1}^{N_{\sigma}} \frac{n_{\sigma i}(\mathbf{r})}{n_{\sigma}(\mathbf{r})}\left(\bar{V}_{x, \sigma i}^{K L I-x}-\bar{u}_{x, \sigma i}\right)
$$

where

$$
V_{x, \sigma}^{S}(\mathbf{r})=\sum_{i=1}^{N_{\sigma}} \frac{n_{\sigma i}(\mathbf{r})}{n_{\sigma}(\mathbf{r})} u_{x, \sigma i}(\mathbf{r})
$$

is the Slater exchange potential, [28] with $n_{\sigma i}(\mathbf{r})=f_{\sigma i}\left|\psi_{\sigma i}(\mathbf{r})\right|^{2}$. The quantities $\bar{V}_{x, \sigma i}^{K L I-x}$ and $\bar{u}_{x, \sigma i}$ are the expectation values of $V_{x}^{K L I-x}(\mathbf{r})$ and $u_{x, \sigma i}(\mathbf{r})$ with respect to the KLI-x orbital $\sigma i$,

$$
\bar{V}_{x, \sigma i}^{K L I-x}=\left\langle\psi_{\sigma i}\left|V_{x}^{K L I-x}\right| \psi_{\sigma i}\right\rangle ; \bar{u}_{x, \sigma i}=\left\langle\psi_{\sigma i}\left|u_{x, \sigma i}\right| \psi_{\sigma i}\right\rangle
$$


The condition

$$
\bar{V}_{x, \sigma N_{\sigma}}^{K L I-x}=\bar{u}_{x, \sigma N_{\sigma}}
$$

is automatically satisfied with the exact exchange energy functional, [23] so only the remaining $N_{\sigma}-1$ constants $\left(\bar{V}_{x, \sigma i}^{K L I-x}-\bar{u}_{x, \sigma i}\right)$ in Eq. (11) need to be determined by the solution of the following linear equation:

$$
\sum_{i=1}^{N_{\sigma}-1}\left(\delta_{j i}-M_{\sigma j i}\right)\left(\bar{V}_{x, \sigma i}^{K L I-x}-\bar{u}_{x, \sigma i}\right)=\left(\bar{V}_{x, \sigma i}^{S}-\bar{u}_{x, \sigma i}\right),
$$

where

$$
M_{\sigma j i} \equiv \int d \mathbf{r} \frac{n_{\sigma j}(\mathbf{r}) n_{\sigma i}(\mathbf{r})}{n_{\sigma}(\mathbf{r})}, i, j=1, \ldots, N_{\sigma}-1
$$

The Slater potential $V_{x, \sigma}^{S}(\mathbf{r})$ in Eq. (11) ensures the correct asymptotic behavior of $-1 / r$ for large $r$ when the exact exchange energy expression is employed. [16,18 It is known that $V_{x, \sigma}^{S}(\mathbf{r})$ alone is too deep, [18] and is a factor of $3 / 2$ larger than $V_{x, \sigma}^{K S}=V_{x, \sigma}^{L S D}$ in the homogeneous-electron-gas limit. The second term in Eq. (11), which was originally

missing in the Slater's proposition, enables $V_{x, \sigma}^{K L I-x}(\mathbf{r})$ to correctly reduce to $V_{x, \sigma}^{L S D}$ in the homogeneous-electron-gas limit, and to preserve the property of the integer discontinuity. 18] Also, due to Eq. (14), the KLI-x highest occupied eigenvalues satisfy the following condition

$$
\begin{aligned}
& \epsilon_{\sigma N_{\sigma}}^{K L I-x}=\left\langle\psi_{\sigma N_{\sigma}}\left|\left(-\frac{1}{2} \nabla^{2}+V_{e x t}+V_{H}+V_{x}^{K L I-x}\right)\right| \psi_{\sigma N_{\sigma}}\right\rangle \\
& =\left\langle\psi_{\sigma N_{\sigma}}\left|\left(-\frac{1}{2} \nabla^{2}+V_{e x t}+V_{H}+\bar{u}_{x, \sigma N_{\sigma}}\right)\right| \psi_{\sigma N_{\sigma}}\right\rangle=\epsilon_{\sigma N_{\sigma}}^{H F^{\prime}}
\end{aligned}
$$

where $\epsilon_{\sigma N_{\sigma}}^{H F^{\prime}}$ is the HF orbital energy expression evaluated with the KS (rather than HF) orbital $\psi_{\sigma N_{\sigma}}$.

At this point, we would like to emphasize that the KLI-x KS scheme yields a local potential, while the HF method gives a nonlocal exchange potential. It is well known that the HF method gives eigenfunctions and eigenvalues for the unoccupied orbitals which are inappropriate for describing actual excited states, because HF unoccupied orbitals do not have self-interaction corrections. [29] This point will be further emphasized and discussed in Sec. IIIB with specific examples.

\section{B. Computational Methods}

All the calculations in this work are based on the finite-difference pseudopotential scheme [26] which we have previously applied to the study of neutral and charged water clusters. 27] In this formulation, quantities such as KS orbitals, densities, and potentials are expressed on grids in a rectangular simulation box, and the Laplacians or gradients are evaluated by higher-order finite-difference expressions. The most computationally demanding step in the construction of the KLI-x potential is the computation of the $\left(N_{\sigma}-1\right) N_{\sigma} / 2$ pairs 
$\int d \mathbf{r}^{\prime} \psi_{\sigma j}^{*}\left(\mathbf{r}^{\prime}\right) \psi_{\sigma i}\left(\mathbf{r}^{\prime}\right) /\left|\mathbf{r}-\mathbf{r}^{\prime}\right|$ in Eq.(9). Since these terms have the same structure as that of the Hartree potential with density $n_{\sigma}\left(\mathbf{r}^{\prime}\right)$ replaced by $\psi_{\sigma j}^{*}\left(\mathbf{r}^{\prime}\right) \psi_{\sigma i}\left(\mathbf{r}^{\prime}\right)$, we use the techniques we have developed for the solution of the Poisson equation, and solve the discretized Poisson-like equations

$$
\nabla^{2} K_{\sigma j i}(\mathbf{r})=-4 \pi \psi_{\sigma j}^{*}(\mathbf{r}) \psi_{\sigma i}(\mathbf{r})
$$

To do so, we first generated boundary values for $K_{\sigma j i}(\mathbf{r})$ at six sides of the simulation box using a multipole expansion. Multipole expansions have been performed until the boundary value error $\left|\delta K_{\sigma j i}\left(\mathbf{r}_{\text {boundary }}\right)\right| /\left|K_{\sigma j i}\left(\mathbf{r}_{\text {boundary }}\right)\right|$ was less than $10^{-3}$. Next, a coarse solution for $K_{\sigma j i}(\mathbf{r})$ on the entire simulation grid has been generated by the fast Fourier transform method using a lower-order (3 points along each direction) finite-difference expression. This solution has been subsequently relaxed by the iterative preconditioned conjugate gradient method using a higher-order (typically 13 points along each direction) finitedifference method. Conjugate gradient relaxation steps have been performed until the error $\left|\delta K_{\sigma j i}(\mathbf{r})\right| /\left|K_{\sigma j i}(\mathbf{r})\right|$ is decreased to below $10^{-3}$. For the preconditioning, a smoothing type preconditioner has been used. We observed that potential mixing rather than density mixing is more appropriate, which should be understandable considering that the KLI-x potential is orbital-dependent.

In addition, for the GGA calculations, we have constructed GGA potentials with the scheme of White and Bird. [30] For other computational details including the implementation of the GGA potential, we refer to our other publications. 27, 31, 32

\section{Test calculations}

To assess the accuracy of our 3D KLI-x implementation, we considered atoms and compared LDA and KLI-x results with those from an accurate 1D radial atomic code. One test was for an Appelbaum-Hamann type local pseudopotential 33 modified to bind 5 electrons $V(r)=-\frac{5}{r} \operatorname{erf}(\sqrt{\alpha} r)+\left(v_{1}+v_{2} r^{2}\right) e^{-\alpha r^{2}}$, where $\alpha=0.6102, v_{1}=3.042$, and $v_{2}=-1.732$. This choice was made to reconcile the limitations of the $3 \mathrm{D}$ code that it can deal with only smooth pseudopotentials and the 1D code that it can only produce accurate results for spherical atoms because of the central field approximation. Using a grid spacing $h=0.4$ a.u., simulation cell length $L=32$ a.u., and finite-difference order 13, we obtained agreement with the radial code of $\leq 10^{-3}$ a.u./atom for both LDA and KLI-x total energies. For the hydrogen atom, we have used a local potential derived by Giannozzi 34 and again obtained an agreement of $\leq 10^{-3}$ a.u./atom using $h=0.4$ a.u. For several other atoms considered in the next section $(\mathrm{C}, \mathrm{N}$, and $\mathrm{O})$, we generated LDA pseudopotentials with the method of Troullier and Martins [35] and compared LDA total energies and eigenvalues from the two codes. With $h=0.25$ a.u., the 3D code reproduced the $1 \mathrm{D}$ atomic total energy with an accuracy of $\leq 5 \times 10^{-3}$ a.u./atom which is sufficient for our purpose.

Before closing this section, we would like to comment on the issues involved with the use of pseudopotentials. Although the rigorous and consistent procedure would be to employ pseudopotentials generated within the same functional approximations, we used LDA generated pseudopotentials to carry out all other functional calculations for two reasons. First, we believe this procedure will not change the qualitative picture, since structural and electronic 
properties are rather insensitive on the nature of the exchange-correlation energy functional that is used for the small 1s cores of the first-row atoms considered here. [36] Second, we note ambiguities associated with pseudopotential generation procedures. For example, we observed a small but sharp peak at the atomic center in the PBE GGA pseudopoential, which caused a serious convergence problem. [32 Moreover, in the KLI-x pseudopotential generation, there is an intrinsic problem of slow decaying tails due to the nonlocal nature of the exact exchange functional that somewhat influences the results. [24, 37] These anomalous behaviors can be only alleviated by some post-processings which introduce additional arbitrariness in pseudopotenials. [24, 32]

\section{RESULTS AND DISCUSSION}

\section{A. Structural properties}

We first examine the structural properties of $\mathrm{H}_{2}, \mathrm{CO}, \mathrm{N}_{2}, \mathrm{H}_{2} \mathrm{O}$, and $\mathrm{CH}_{4}$ given in Table —and Table [1]. The binding energies $E_{B}$, equilibrium bond lengths $r_{e}$, and vibrational frequencies $\omega_{e}$ of the diatomic molecules were determined from the total energy versus bondlength curves by fitting to the five parameter $\left(D_{e}, r_{e}, \omega_{e}\right.$, a, and b) Hulburt-Hirschfelder function, 38,39

$$
V(r)=D_{e}\left[\left(1-e^{-\beta x}\right)^{2}+b \beta^{3} x^{3} e^{-2 \beta x}(1+a \beta x)-1\right],
$$

where $x=r-r_{e}$ and $r_{e}$ and $D_{e}$ are the equilibrium distance and bonding energy, using the simulated annealing method. [40] The geometries of the polyatomic molecules have been optimized by employing Hellmann-Feynman quantum forces. A grid spacing $h=0.25$ a.u. has been used for all the molecular calculations. For the calculation of binding energies, the energies of each pseudoatom and molecules have been calculated by the same method and with the same $h$. We expect this procedure will result in a systematic cancellation of errors, and in addition we may treat the atom without central field approximation.

Our LDA and GGA results are essentially a reproduction of previous studies. 411 43] The LDA consistently overestimates the stability of the molecules and the GGA typically cures this tendency of overestimation to a large extent. The HF approximation on the other hand substantially underestimates the binding energy. 39,44 Bond lengths calculated by the LDA are usually overestimated and one obtains corresponding underestimations of vibrational frequencies for the diatomic molecules. The HF method gives the opposite behavior. The KLI-x approximation typically gives similar results as HF for the structural properties which are mostly determined by the total energy functional. [44] It is well known that the HF method gives the wrong sign of the dipole moment of $C O$, and the LDA corrects this. Again, the KLI-x calculation yields the wrong sign of the dipole moment of $C O$ in agreement with the HF method. The difference between magnitudes of the KLI-x and HF dipole moment of $C O$ results from the fact that the $\mathrm{HF}$ value has been evaluated at the experimental bond length. Our KLI-x calculation at the experimental bond length gave -0.275 Debye.

The addition of an LDA or GGA correlation energy functional to the exact exchange energy gives mixed results: Binding energies are increased, hence improved the KLI-x values, 
but bond lengths are decreased and become worse. So, we can conclude that the two correlation functional approximations do not give systematic improvements over the exact exchange energy functional, which indicates that these approximations are not accurate descriptions of correlation effects in molecules.

\section{B. KS Eigenvalues and Excitations}

In Fig. 1, we compare the LDA-xc and KLI-x potentials of $H_{2}$ to reemphasize the different nature of the exchange(-correlation) potentials from the two methods. While the LDA-xc potential decays exponentially, the KLI-x potential reproduces the correct $-1 / r$ asymptotic behavior, and this causes marked differences in the eigenvalue spectra. This $-1 / r$ decay property lets the high-lying KS unoccupied eigenvalues correctly approach a Rydberg series, and is also important for other properties that are sensitive to the outer part of the charge density, such as polarizabilities.

Eigenvalues of the highest occupied orbitals of the molecules considered in the previous section at their equilibrium geometries (Tables II and III) are listed in Table III. Compared with the LDA or GGA, the KLI-x values agree very well with experimental ionization potentials, and they are very close to HF values. It is well known that HF gives a good approximation to the ionization potential via Koopman's theorem due to the fact that omission of correlation tends to be cancelled by the neglect of relaxation in the "frozen orbital" approximation. 444 On the other hand, in the exact DFT, the highest occupied orbital eigenvalue equals the ionization potential without any relaxation correction [10,12] as we typically see in our KLI-x calculations, hence, in principle DFT should yield highest occupied orbital eigenvalues in better agreement with experimental ionization potentials than HF. However, in the usual LDA or GGA, the highest occupied eigenvalues are in serious errors due to the wrong decay property of exchange-correlation potential exemplified in Fig. 1. The errors are about $0.2 \mathrm{a}$.u. for the molecules we have studied as shown in Table III. On the other hand, due to Eq. (17), KLI-x should give highest occupied orbital eigenvalues in good agreement with experimental ionization potentials when HF values also agree with experimental ionization potential. The addition of LDA or GGA correlation potentials to the KLI-x potential usually lowers KLI-x highest occupied orbital eigenvalues, but this leads to too large ionization potentials.

Among the molecules examined in this work, $N_{2}$ is a particularly interesting case which shows the advantage and disadvantage of the KLI-x scheme at the same time. First, note that the HF results are in qualitative disagreement with experiment: it puts the $1 \pi_{u}$ state higher than $3 \sigma_{g}$ state as the highest occupied orbital in contrast to the experimental data. This incorrect ordering of the first two ionization potentials is a well-known example of the breakdown of the HF picture and indicates a large effect of correlation upon the orbitals in $N_{2}$. 44 On the other hand note that KLI-x gives the correct ordering of the two orbitals, which implies that the local nature of the KLI-x potential can make a difference even for the occupied-orbital eigenvalue spectrum. The fact that the LDA also gives the correct ordering (although the value is worse than the KLI-x one due to the above-mentioned reasons) shows that there are cases where the local potential in the KS scheme is superior to the nonlocal

HF potential. The highest occupied state in the KLI-x scheme $\epsilon_{3 \sigma_{g}}^{K L I-x}$ is equal to $\epsilon_{3 \sigma_{g}}^{H F^{\prime}}$ [according to Eq. (17)] which results in the biggest KLI-x error among the molecules studied 
since this also has the largest HF error. Better quantitative agreement with experiment can be obtained by incorporating the correlations, e.g. through many-body perturbation type approach. 44

In addition to the eigenvalues of the highest occupied orbitals, we have also examined eigenvalue differences between the highest occupied and the lowest unoccupied orbitals, which are compared with experimental lowest triplet and singlet vertical excitation energies in Table IV. First, we point out that the HF calculations give positive orbital energies for all the virtual orbitals in the molecules considered here. [39,44] This is because unoccupied orbitals in HF do not really correspond to excited states of the system, in which the excited state electron would have been removed from one of the lower states and acted on by $N-1$ remaining electrons, [29] but rather the bound states (if any) of the singly-charged negative ion, in which the extra electron sees the field due to other $N$ electrons. [39 To make this argument more explicit, we follow Slater [45 and rewrite Eqs. (9) and (10) (now using the HF orbitals $\psi_{\sigma i}^{H F}$ instead of KS orbitals $\psi_{\sigma i}$, so $u_{x, \sigma i}^{H F}$ is the true HF local exchange potential) as

$$
u_{x, \sigma i}^{H F}(\mathbf{r})=-\int d \mathbf{r}^{\prime} \frac{\left[\sum_{j=1}^{N_{\sigma}} f_{\sigma j} \psi_{\sigma i}^{H F *}(\mathbf{r}) \psi_{\sigma j}^{H F *}\left(\mathbf{r}^{\prime}\right) \psi_{\sigma j}^{H F}(\mathbf{r}) \psi_{\sigma i}^{H F}\left(\mathbf{r}^{\prime}\right)\right] /\left[\psi_{\sigma i}^{H F *}(\mathbf{r}) \psi_{\sigma i}^{H F}(\mathbf{r})\right]}{\left|\mathbf{r}-\mathbf{r}^{\prime}\right|},
$$

and identify

$$
-\frac{\sum_{j=1}^{N_{\sigma}} f_{\sigma j} \psi_{\sigma i}^{H F *}(\mathbf{r}) \psi_{\sigma j}^{H F *}\left(\mathbf{r}^{\prime}\right) \psi_{\sigma j}^{H F}(\mathbf{r}) \psi_{\sigma i}^{H F}\left(\mathbf{r}^{\prime}\right)}{\psi_{\sigma i}^{H F *}(\mathbf{r}) \psi_{\sigma i}^{H F}(\mathbf{r})}
$$

as an exchange charge density. Because the $\psi_{\sigma i}^{H F}$ 's are orthonormal, the exchange charge density integrated over $d \mathbf{r}^{\prime}$ will be minus one for the occupied orbital $(j=i$ term exists in the summation $\sum_{j=1}^{N_{\sigma}}$ and $\left.f_{\sigma i}=1\right)$, but zero for the unoccupied orbital $\left(\psi_{\sigma i}^{H F}\right.$ is not included in $\left.\sum_{j=1}^{N_{\sigma}}\right)$. Hence, the self-consistent field in which an unoccupied orbital moves is that of the nucleus and all $N$ electrons instead of $N-1$ electrons.

On the other hand, in DFT, as mentioned in Sec. [1, differences of KS eigenvalues are welldefined approximations for excitation energies. [7] Umrigar et al. have confirmed that this is the case for several atomic systems by showing that the exact $\mathrm{KS}$ eigenvalue differences obtained from KS potentials derived from accurate QMC densities almost always lie between the triplet and singlet experimental excitation energies. [8] They further claimed that this is because of the fact that, in addition to the well known exact properties of $V_{x c}^{K S}$ (Sec. 回), $V_{x c}^{K S}$ agrees with the quasiparticle amplitude [10] for $r \rightarrow \infty$ not only up to $1 / r$ but up to order $1 / r^{4}$ inclusive. However, in the LDA or GGA, no bound unoccupied state exists in many cases, due to the shallow nature of the corresponding exchange-correlation potentials. We can expect that the KLI-x is a much better approximation than the LDA/GGA, since it satisfies Eq. (17) and has the correct asymptotic $-1 / r$ behavior. Our KLI-x results indeed always give negative virtual orbital eigenvalues for the studied molecules and also show good agreement with experimental values. The single exception is $\mathrm{H}_{2} \mathrm{O}$ in which the difference between the highest occupied and lowest unoccupied orbital eigenvalues is bigger than the experimental triplet and singlet excitation energies by $\approx 0.07$ a.u. Further detailed agreements with different multiplet states can be obtained by employing a more involved theory such as DFT perturbation theory [7,9] or time-dependent DFT. [5, 6] 
We close this section by reconsidering the meaning of eigenvalues in the OEP-x (or KLI$\mathrm{x}$ ) and HF schemes to make the differences between the two be clear. In the fractional occupation number formalism, KLI have shown that

$$
\frac{\partial E}{\partial f_{i}}=\left\langle\psi_{\sigma i}\left|\left(-\frac{1}{2} \nabla^{2}+V_{e x t}+V_{H}+u_{x, \sigma i}\right)\right| \psi_{\sigma i}\right\rangle \equiv \epsilon_{\sigma i}^{H F^{\prime}},
$$

where $\epsilon_{\sigma i}^{H F^{\prime}}$ is the HF orbital energy expression for the orbital $\sigma i$ evaluated using the OEP-x orbitals $\psi_{\sigma i}$. [18] We note that this can be generalized to the correlated OEP case. [18,46] Equation (22) can be rearranged to the form in which the relationship between the two methods is transparent:

$$
\epsilon_{\sigma i}^{O E P-x}+\left\langle\psi_{\sigma i}\left|\left(u_{x, \sigma i}-V_{x}^{O E P-x}\right)\right| \psi_{\sigma i}\right\rangle=\epsilon_{\sigma i}^{H F^{\prime}} .
$$

Note that from the condition $\bar{V}_{x, \sigma N_{\sigma}}^{O E P-x}=\bar{u}_{x, \sigma N_{\sigma}}$, satisfied by both OEP-x and KLI-x [Eq. (14)], Eq. (17) is reproduced from Eq. (23) for the $\sigma N_{\sigma}$ state. Hence, the eigenvalue difference between the highest occupied orbital state $\psi_{\sigma N_{\sigma}}$ and the unoccupied orbital state $\psi_{\sigma a}$ is given by

$$
\begin{array}{r}
\epsilon_{\sigma a}^{O E P-x}-\epsilon_{\sigma N_{\sigma}}^{O E P-x}+\left\langle\psi_{\sigma a}\right|\left(u_{x, \sigma a}-\right. \\
\left.=V_{x}^{O E P-x}\right)\left|\psi_{\sigma a}\right\rangle \\
=\epsilon_{\sigma a}^{H F^{\prime}}-\epsilon_{\sigma N_{\sigma}}^{H F^{\prime}} .
\end{array}
$$

Assuming that the differences between the HF and OEP-x orbitals are negligible, we can see that HF orbital eigenvalue differences are much bigger than OEP-x values, because the $\left\langle\psi_{\sigma a}\left|u_{x, \sigma a}\right| \psi_{\sigma a}\right\rangle$ term is small due to the property of the exchange charge density [Eq. (21)] for the unoccupied orbital as discussed above, while the $\left\langle\psi_{\sigma a}\left|V_{x}^{O E P-x}\right| \psi_{\sigma a}\right\rangle$ term is clearly quite large.

\section{CONCLUSIONS}

In this work, we have studied structural and excitation properties of small molecules using the KLI approximation to the OEP with the exact exchange energy functional only (KLI-x) and augmented by LDA and GGA correlation functionals. For structural properties, the KLI-x gave comparable results to those of the HF method. For excitation properties, the KLI-x results in good eigenvalue spectra for both the highest occupied and unoccupied orbitals, since it has the correct asymptotic large $r$ behavior of $V_{x c}^{K S}$ unlike the LDA or GGA. Especially, we find that unoccupied orbital eigenvalues are better described by KLI-x than by $\mathrm{HF}$, which illustrates the advantage of the KS scheme in general.

\section{ACKNOWLEDGMENTS}

This work has been supported by the National Science Foundation under Grant No. DMR 98-0273 (Y.-H. K. and R. M. M.) and by the Office of Naval Research under Grant No. N0014-98-1-0594 (M. S.). We acknowledge Profs. J. B. Krieger and J. P. Perdew for critical readings, Prof. E. K. U. Gross for helpful discussions, Prof. J. Soler for providing us with his GGA exchange-correlation routine, and Dr. D. Sanchez-Portal for performing GGA calculations for comparisons. 


\section{REFERENCES}

[1] P. Hohenberg and W. Kohn, Phys. Rev. 136, B864 (1964).

[2] W. Kohn and L. J. Sham, Phys. Rev. 140, A1133 (1965).

[3] R. G. Parr and W. Yang, Density Functional Theory of Atoms and Molecules (Oxford University Press, New York, 1989).

[4] A. Görling and M. Levy, Int. J. Quantum Chem. Symp. 29, 93 (1995).

[5] E. K. U. Gross, J. F. Dobson, and M. Petersilka, in Density Functional Theory, edited by R. F. Nalewajski (Springer-Verlag, Berlin, 1996).

[6] M. E. Casida, in Recent Developments and Application of Modern Density Functional Theory, edited by J. M. Seminario (Elsevier, Amsterdam, 1996).

[7] A. Görling, Phys. Rev. A 54, 3912 (1996).

[8] C. J. Umrigar, A. Savin, and X. Gonze, in Electronic Density Functional Thoery: Recent Progress and New Directions, edited by J. F. Dobson, G. Vignale, and M. P. Das (Plenum, New York, 1998).

[9] C. Filippi, C. J. Umrigar, and X. Gonze, J. Chem. Phys. 107, 9994 (1997).

[10] C.-O. Almbladh and U. von Barth, Phys. Rev. B 31, 3231 (1985).

[11] J. P. Perdew and M. Levy, in Many-Body Phenomena at Surfaces, edited by D. C. Langreth and H. Suhl (Acedemic, New York, 1984).

[12] J. P. Perdew, R. G. Parr, M. Levy, and J. L. Balduz, Jr., Phys. Rev. Lett. 49, 1691 (1982).

[13] J. P. Perdew and M. Levy, Phys. Rev. Lett. 51, 1884 (1983).

[14] L. J. Sham and M. Schlüter, Phys. Rev. Lett. 51, 1888 (1983).

[15] R. T. Sharp and G. K. Horton, Phys. Rev. 90, 317 (1953).

[16] J. D. Talman and W. F. Shadwick, Phys. Rev. A 14, 36 (1976).

[17] M. R. Norman and D. D. Koelling, Phys. Rev. B 30, 5530 (1984).

[18] J. B. Krieger, Y. Li, and G. J. Iafrate, Phys. Lett. A 148, 470 (1990); Phys. Rev. A 45, 101 (1992); in Density Functional Thoery, edited by E. K. U. Gross and R. M. Dreizler, 191 (1995).

[19] E. Engel and S. H. Vosko, Phys. Rev. A 47, 2800 (1993).

[20] E. Krotscheck, W. Kohn, and G.-X. Qian, Phys. Rev. B 32, 5693 (1985).

[21] A. Bulgac, C. Lewenkopf, and V. Mickrjukov, Phys. Rev. B 52, 16476 (1995); E. Engel (private communication).

[22] M. Städele, J. A. Majewski, P. Vogl, and A. Görling, Phys. Rev. Lett. 79, 2089 (1997); M. Städele, M. Moukara, J. A. Majewski, P. Vogl, and A. Görling, Phys. Rev. B 59, 10031 (1999).

[23] T. Grabo, T. Kreibich, S. Kurth, and E. K. U. Gross, in Strong Coulomb Correlations in Electronic Structure: Beyond the Local Density Approximation, edited by V. I. Anisimov (Gordon \& Breach, Tokyo, 1998), and references therein.

[24] D. M. Bylander and L. Kleinman, Phys. Rev. Lett. 74, 3660 (1995); Phys. Rev. B 52, 14566 (1995); Phys. Rev. B 54, 7891 (1996); Phys. Rev. B 55, 9432 (1997).

[25] J. P. Perdew, K. Burke, and M. Ernzerhof, Phys. Rev. Lett. 77, 3865 (1996); 78, 1396(E) (1997).

[26] J. R. Chelikowsky, N. Troullier, and Y. Saad, Phys. Rev. Lett. 72, 1240 (1994); J.R. Chelikowsky, N. Troullier, K. Wu and Y. Saad, Phys. Rev. B 50, 11355 (1994).

[27] Y.-H. Kim, I.-H. Lee, and R. M. Martin (in preparation). 
[28] J. C. Slater, Phys. Rev. 81, 385 (1951).

[29] J. C. Slater, J. B. Mann, T. M. Wilson, and J. H. Wood, Phys. Rev. 184, 672 (1969).

[30] J. A. White and D. M. Bird, Phys. Rev. B 50, 4954 (1994).

[31] I.-H. Lee, V. Rao, R. M. Martin, and J.-P. Leburton, Phys. Rev. B 57, 9035 (1998).

[32] I.-H. Lee and R. M. Martin, Phys. Rev. B 56, 7197 (1997).

[33] J. A. Appelbaum and D. R. Hamann, Phys. Rev. B 8, 1777 (1973).

[34] F. Gygi, Phys. Rev. B 48, 11692 (1993).

[35] N. Troullier and J. L. Martins, Phys. Rev. B 43, 1993 (1991).

[36] C. Woodward and A. B. Kunz, Phys. Rev. B 37, 2674 (1988).

[37] M. Städele, Ph. D. thesis, TU München (1998).

[38] H. M. Hulburt and J. O. Hirschfelder, J. Chem. Phys. 61, 61 (1941).

[39] A. C. Hurley, Introduction to the Electronic Theory of Small Molecules (Academic Press, London, 1976).

[40] W. H. Press, S. A. Teukolsky, W. T. Vetterling, and B. P. Flannery, Numerical Recipes in Fortran, 2nd edition (Cambridge University Press, Cambridge, England, 1992).

[41] G. S. Painter and F. W. Averill, Phys. Rev. B 26, 1781 (1982).

[42] F. W. Kutzler and G. S. Painter, Phys. Rev. B 45, 3236 (1992).

[43] D. C. Patton, D. V. Porezag, and M. R. Pederson, Phys. Rev. B 55, 7454 (1997).

[44] A. Szabo and N. S. Ostlund, Modern Quantum Chemistry (McGraw-Hill, New York, 1989), and references therein.

[45] J. C. Slater, Quantum Theory of Molecules and Solids (McGraw-Hill, New York, 1963) Vol. 2.

[46] M. E. Casida, Phys. Rev. B 59, 4694 (1999). 


\section{TABLES}

TABLE I. Binding energies, bond lengths, and vibrational frequencies of $H_{2}, C O$, and $N_{2}$, calculated with different methods. In addition, the dipole moment of $C O$ has been given, whose sign has been defined such that $\mathrm{C}^{-} \mathrm{O}^{+}$is positive. Energies and bond lengths are given in a.u., vibrational frequencies in $\mathrm{cm}^{-1}$, and dipole moments in Debye.

\begin{tabular}{lcccccccccc}
\hline \hline & \multicolumn{3}{c}{$H_{2}$} & \multicolumn{1}{c}{$C O$} & \multicolumn{3}{c}{$N_{2}$} \\
& $E_{B}$ & $r_{e}$ & $\omega_{e}$ & $E_{B}$ & $r_{e}$ & $\omega_{e}$ & $\mu$ & $E_{B}$ & $r_{e}$ & $\omega_{e}$ \\
\hline LDA-xc & 0.184 & 1.44 & 4228 & 0.466 & 2.13 & 2193 & 0.248 & 0.396 & 2.09 & 2385 \\
GGA-xc & 0.168 & 1.41 & 4228 & 0.424 & 2.12 & 2166 & 0.265 & 0.368 & 2.09 & 2383 \\
\hline KLI-x & 0.136 & 1.39 & 4647 & 0.287 & 2.06 & 2445 & -0.163 & 0.176 & 2.01 & 2634 \\
+LDA-c & 0.185 & 1.36 & 4765 & 0.342 & 2.05 & 2595 & -0.184 & 0.271 & 2.00 & 2710 \\
+GGA-c $^{\text {GG-a }}$ & 0.166 & 1.38 & 4734 & 0.361 & 2.06 & 2641 & -0.170 & 0.273 & 2.00 & 2805 \\
\hline HF-x $^{\text {a }}$ & 0.134 & 1.39 & 4582 & 0.293 & 2.08 & 2431 & $-0.279^{b}$ & 0.195 & 2.01 & 2730 \\
\hline Exp. & 0.174 & 1.40 & 4400 & 0.414 & 2.13 & 2170 & 0.123 & 0.364 & 2.07 & 2358 \\
\hline \hline
\end{tabular}

a Reference [39].

${ }^{\mathrm{b}}$ At the experimental bond length.

TABLE II. Binding energies and bond lengths of $\mathrm{CH}_{4}$ and $\mathrm{H}_{2} \mathrm{O}$, and the bond angle of $\mathrm{H}_{2} \mathrm{O}$. Binding energies and bond lengths are given in a.u., bond angles are given in degrees.

\begin{tabular}{llclcc}
\hline \hline & \multicolumn{2}{c}{$\mathrm{CH}_{4}$} & \multicolumn{3}{c}{$\mathrm{H}_{2} \mathrm{O}$} \\
& $E_{b}$ & $r_{e}(C-H)$ & $E_{b}$ & $r_{e}(O-H)$ & $\theta(H-O-H)$ \\
\hline LDA-xc & 0.743 & 2.07 & 0.420 & 1.82 & 104.2 \\
GGA-xc & 0.681 & 2.06 & 0.370 & 1.81 & 104.5 \\
\hline KLI-x & 0.536 & 2.05 & 0.257 & 1.76 & 106.0 \\
+ LDA-c & 0.678 & 2.04 & 0.344 & 1.76 & 106.0 \\
+GGA-c $_{\text {HF-x }}^{\text {a }}$ & 0.672 & 2.03 & 0.339 & 1.77 & 105.3 \\
\hline Exp. $^{\text {a }}$ & 0.522 & 2.05 & 0.258 & 1.78 & 106.1 \\
\hline \hline
\end{tabular}

${ }^{a}$ Reference [39]. 
TABLE III. Absolute values of highest occupied orbital eigenvalues of the $\mathrm{H}_{2}, \mathrm{CO}, \mathrm{N}_{2}, \mathrm{H}_{2} \mathrm{O}$, and $\mathrm{CH}_{4}$ at their equilibrium geometries (Table I and II). For $N_{2}$, absolute values of the next highest occupied orbital eigenvalues are also given. Note the differet ordering of HF and KLI-x for this case. Experimental values are ionization potentials. Energies are in a.u.

\begin{tabular}{lccccc}
\hline \hline & $H_{2}\left(-\epsilon_{1 \sigma_{g}}\right)$ & $C O\left(-\epsilon_{5 \sigma}\right)$ & $N_{2}\left(-\epsilon_{3 \sigma_{g}} /-\epsilon_{1 \pi_{u}}\right)$ & $H_{2} O\left(-\epsilon_{1 b_{1}}\right)$ & $C_{4}\left(-\epsilon_{1 t_{2}}\right)$ \\
\hline LDA-xc & 0.37 & 0.34 & $0.38 / 0.44$ & 0.26 & 0.34 \\
GGA-xc & 0.38 & 0.34 & $0.38 / 0.43$ & 0.27 & 0.34 \\
\hline KLI-x & 0.60 & 0.55 & $0.64 / 0.69$ & 0.50 & 0.54 \\
KLI-x + LDA-c & 0.60 & 0.61 & $0.69 / 0.75$ & 0.56 & 0.60 \\
KLI-x + GGA-c & 0.58 & 0.59 & $0.67 / 0.74$ & 0.55 & 0.58 \\
\hline HF-x $^{\text {a }}$ & 0.60 & 0.55 & $0.64 / 0.62$ & 0.51 & 0.55 \\
\hline Exp. $^{\text {a }}$ & 0.58 & 0.58 & $0.57 / 0.62$ & 0.46 & 0.53 \\
\hline
\end{tabular}

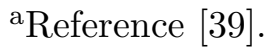

TABLE IV. Differences between highest occupied and lowest unoccupied orbital states eigenvalues of $\mathrm{H}_{2}, \mathrm{CO}, \mathrm{N}_{2}, \mathrm{H}_{2} \mathrm{O}$, and $\mathrm{CH}_{4}$ at their respective equilibrium geometries given in Table I and II. A - mark indicates that the lowest unoccupied orbital state is in the continuum and hence unstable. Experimental values are vertical excitation energies to final triplet and singlet states shown in parentheses. Energies are in a.u.

\begin{tabular}{lccccc}
\hline \hline & $H_{2}\left(\epsilon_{1 \sigma_{u}}-\epsilon_{1 \sigma_{g}}\right)$ & $C O\left(\epsilon_{2 \pi}-\epsilon_{5 \sigma}\right)$ & $N_{2}\left(\epsilon_{1 \pi_{g}}-\epsilon_{3 \sigma_{g}}\right)$ & $H_{2} O\left(\epsilon_{4 a_{1}}-\epsilon_{1 b_{1}}\right) C_{4}\left(\epsilon_{3 a_{1}}-\epsilon_{1 t_{2}}\right)$ \\
\hline LDA-xc & - & 0.26 & 0.30 & - & - \\
GGA-xc & - & 0.26 & 0.31 & - & - \\
\hline KLI-x & 0.47 & 0.28 & 0.34 & 0.34 & 0.42 \\
KLI-x + LDA-c & 0.50 & 0.28 & 0.34 & 0.36 & 0.46 \\
KLI-x + GGA-c & 0.49 & 0.27 & 0.34 & 0.37 & 0.45 \\
\hline HF-x & - & - & - & - & - \\
\hline Exp. ${ }^{\text {a }}$ & $0.42\left({ }^{3} \Sigma_{u}^{+}\right)$ & $0.23\left({ }^{3} \Pi\right)$ & $0.28\left({ }^{3} \Sigma_{u}^{+}\right)$ & $0.26\left({ }^{3} B_{1}\right)$ & $0.40\left({ }^{3} T_{2}\right)$ \\
& $0.46\left({ }^{1} \Pi_{u}\right)$ & $0.31\left({ }^{1} \Pi\right)$ & $0.34\left({ }^{1} \Pi_{g}\right)$ & $0.28\left({ }^{1} B_{1}\right)$ & $0.41\left({ }^{1} T_{2}\right)$ \\
\hline \hline
\end{tabular}

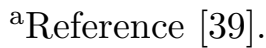




\section{FIGURES}

FIG. 1. Comparison of the LDA exchange-correlation potential and the KLI exchange potential

for $H_{2}$ with $H$ atoms located at \pm 0.7 a.u. Note that the LDA exchange-correlation potential incorrectly decays exponentially, while the KLI exchange potential decays with $-1 / r$ as in the exact Kohn-Sham exchange-correlation potential. 


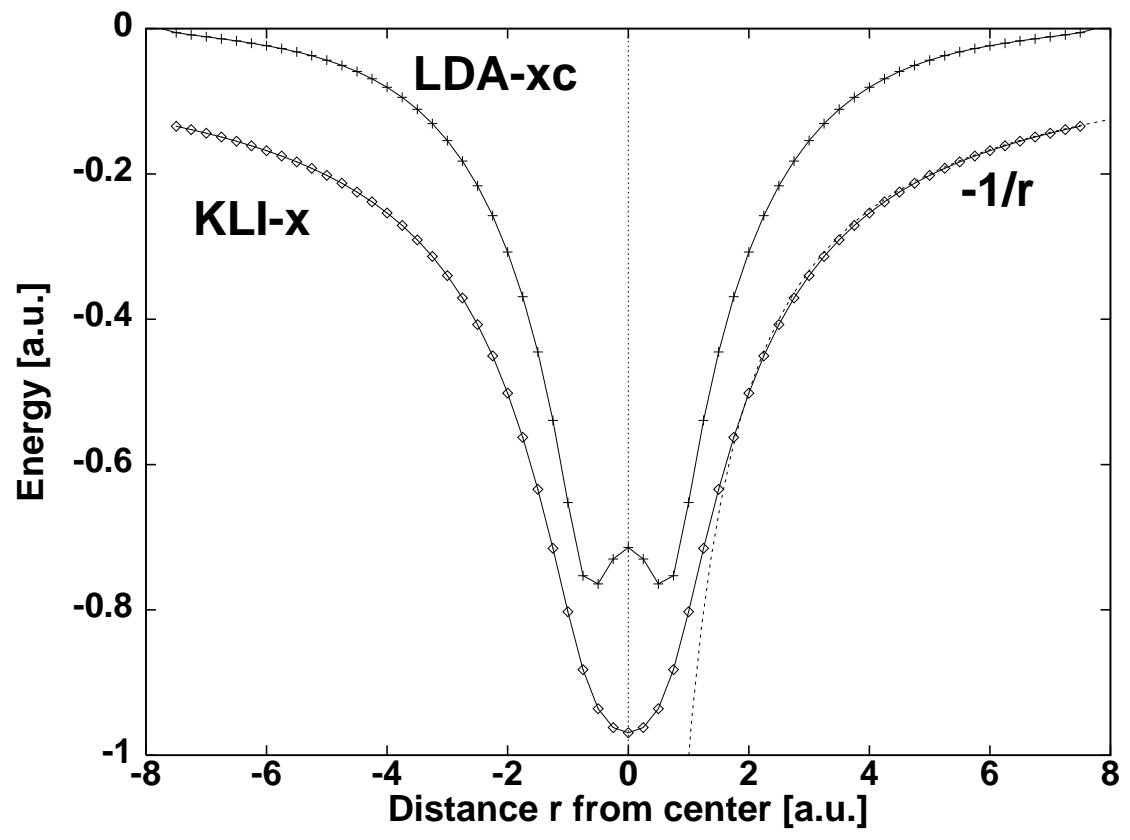

Fig. 1 